\title{
Au fondement des sociétés humaines. Ce que nous apprend l'anthropologie de Maurice Godelier
}

\section{Gilles Bounoure}

\section{OpenEdition}

1 Journals

Édition électronique

URL : http://journals.openedition.org/jso/2062

DOI : $10.4000 /$ jso.2062

ISSN : $1760-7256$

Éditeur

Société des océanistes

Édition imprimée

Date de publication : 15 décembre 2008

Pagination : $339-341$

ISBN : 978-2-85430-012-3

ISSN : 0300-953x

Référence électronique

Gilles Bounoure, "Au fondement des sociétés humaines. Ce que nous apprend l'anthropologie de Maurice Godelier », Journal de la Société des Océanistes [En ligne], 126-127| Année 2008, mis en ligne le 01

décembre 2008, consulté le 21 septembre 2020. URL : http://journals.openedition.org/jso/2062 ; DOI : https://doi.org/10.4000/jso.2062

Ce document a été généré automatiquement le 21 septembre 2020.

(c) Tous droits réservés 


\title{
Au fondement des sociétés humaines. Ce que nous apprend l'anthropologie de Maurice Godelier
}

\author{
Gilles Bounoure
}

\section{RÉFÉRENCE}

Maurice GODELIER, Au fondement des sociétés humaines. Ce que nous apprend l'anthropologie, Paris, Albin Michel, coll. Bibliothèque Idées, 300 p., bibliographie, quadruple index.

1 Sans chercher présomptueusement à discuter un ouvrage appelé à faire date par le prestige de son auteur, l'importance des propositions qu'il y formule et les débats qu'elles ne manqueront pas de susciter, il faut au moins présenter rapidement ce qui peut y concerner directement les Océanistes, forcément intéressés par les travaux de Maurice Godelier, président de notre société. D'abord, comme il est trop rare en pareille matière, c'est un livre qu'on peut lire d'une traite, ou en deux bonnes soirées en prenant des notes, et qui présente avec une remarquable clarté les principaux enjeux passés et actuels de l'anthropologie, à l'attention des lecteurs spécialisés ou non. Sa publication chez un éditeur s'adressant de préférence au " grand public » trouve une parfaite justification dans les dons d'exposition et la hauteur de vues qui s'y déploient, de façon non plus seulement magistrale mais souveraine. Tous les Océanistes, anthropologues ou non, familiers ou non de l'œuvre de Maurice Godelier, pourront aussi y prendre la mesure de près de cinquante ans de recherche, de réflexion et d'enseignement, dont ce livre présente moins un bilan rétrospectif que les conclusions présentes, avec leurs conséquences immédiatement applicables et leurs suites prospectives.

2 Comme l'indique le titre, le propos est double, ou s'articule plus exactement en deux volets inséparables d'argumentations qu'on pourrait dire «théoriques» et de démonstrations «concrètes» ou par l'exemple. La «défense et illustration» de 
l'anthropologie qui occupe explicitement l'introduction et la conclusion (À quoi sert l'anthropologie? pp. 7-64, Éloge des sciences sociales, pp. 221-248), et implicitement tous les chapitres intermédiaires, montre comment cette discipline se trouve injustement contestée de l'intérieur même du monde savant, par les abus de l'hypercritique, l'inflation du "politiquement correct» ou l'hégémonisme d'autres disciplines censément plus "scientifiques", à l'heure où elle est aussi menacée de l'extérieur, en même temps que toutes les sciences sociales identiquement vidées de leur objet, par les politiciens partageant cette vue prétendument «pragmatique » de Margaret Thatcher :

« There is no such thing as society, there are individual men and women, and there are families.» (Aids, Education and the Year 2000, Woman's Own, 31 octobre 1987, p. 10)

3 Sans aborder ouvertement ce dernier aspect qui pèse lourd dans l'actuelle «crise de l'anthropologie ", Maurice Godelier démontre de façon vigoureuse et convaincante l'utilité présente et présumablement future de sciences sociales seules susceptibles d'éclairer dans leur logique profonde des événements aussi décisifs et énigmatiques que les attentats du 11 septembre 2001, auxquels il consacre des développements passionnants (pp. 231-247).

4 Science qu'on pourrait dire «citoyenne " (et à la portée de tout citoyen qui veut s'y intéresser, il est réconfortant de le vérifier ici), dont aucune démocratie moderne et réellement soucieuse de ne pas dégénérer ne saurait prétendre se passer comme d'un "luxe inutile ", l'anthropologie se définit aussi elle-même comme science dans son exercice propre, qui est d'observer, d'établir et de rassembler des faits, de les interpréter, de les comparer, et de soumettre tous ses résultats à l'épreuve de la discussion, aussi bien qu'à l'éclairage de découvertes ou de théories nouvelles. Pour administrer la preuve qu'à l'instar des autres disciplines scientifiques - dont certains représentants lui dénient sous ce prétexte ou d'autres sa «scientificité " l'anthropologie est une science vivante, et même sur des « objets d'étude » ayant cessé de vivre, sociétés et individualités aux spécificités submergées par l'expansion occidentale, tels les Baruya désormais aux marges de la «mondialisation», Maurice Godelier prend comme cas d'école ses propres travaux, «de terrain» autant que «de laboratoire ", et plus que leur détail, les principaux «sauts qualitatifs » qui les ont marqués, pour se détacher et s'affranchir de plus en plus des théories dominantes. L'Océanie occupe ainsi plus de la moitié du livre, et non pas les seuls Baruya et autres sociétés de Nouvelle-Guinée, mais les Maenge, les Trobriandais, les Maori de NouvelleZélande, les Tongiens, les Tikopiens, dont les conceptions sociales ne perdent certainement rien en dignité à se trouver comparées à celles de l'Égypte pharaonique ou de la Chine impériale.

5 Les six chapitres formant le corps de l'ouvrage s'ouvrent sur des titres frappants, en majorité assertoriques, et qu'il faut tous citer : 1. Des choses que l'on donne, des choses que l'on vend et de celles qu'il ne faut ni vendre ni donner mais garder pour les transmettre ; 2 . Nulle société n'a jamais été fondée sur la famille ou sur la parenté ; 3 . Il faut toujours plus qu'un homme et une femme pour faire un enfant; 4. La sexualité humaine est fondamentalement a-sociale ; 5 . Comment un individu se constitue en sujet social; 6. Comment des groupes humains se constituent en société. De ces développements brillamment enchaînés comme autant de leçons ou de parties d'un discours constamment dominé du même souffle - plusieurs viennent de conférences récentes, mais le talent de l'auteur est encore plus manifeste à observer comme il les a 
reformulées, en orateur autant qu'en théoricien -, il résulte que le «fondement des sociétés humaines " n'est plus à chercher dans ce qu'ont cru en comprendre les marxistes, ni les structuralistes, ni d'autres «écoles» un peu moins «systématiques». Leur réfutation aboutit à la conclusion que les êtres humains ne s'agrègent en société que pour autant qu'ils éprouvent en commun « du sacré », donnant lieu à la création ou à l'invention de ces complexes "politico-religieux» qui, selon ce livre, forment le ressort ou le ciment «idéel » (et non plus «matériel » ou «familial ») des sociétés, et les différencient les unes des autres.

6 «Du sacré », mais qui n'est pas tout le sacré (supposé qu'un anthropologue ou quelque autre être humain puisse le définir aujourd'hui), «du politico-religieux", mais qui renvoie sommairement à tout ce qu'a vécu l'espèce humaine d'expériences analogues rapportées par les historiens ou les anthropologues, la brièveté de ce livre empêchait évidemment d'inventorier tout ce que peuvent ou doivent subsumer ces expressions. De même, les lecteurs avertis s'étonneront de voir la question de la famille et de la parenté "expédiée » en si peu de pages (chapitre 2, pp.91-114), plus nombreuses pourtant que celles rapidement consacrées à la constitution du sujet social, notamment à la lumière de la psychanalyse (chapitre $5, \mathrm{pp}$. 177-190). Sur ces sujets comme sur le sacré individuel ou collectif, ou même les vues de Leiris ou Bataille à ce propos (pour citer des références alimentant nombre de réflexions actuelles), ces mêmes lecteurs devront accepter de se reporter aux développements conséquents qu'y a consacrés Maurice Godelier plus ou moins récemment - et pour commencer aux 678 pages des Métamorphoses de la parenté (Paris, Fayard, 2004). Le grand public regrettera peut-être que la bibliographie le dirige vers des travaux en langue étrangère sans mentionner leurs éditions françaises (Frankfort, Malinowski, Weiner, etc. ; seule exception, Mead), mais il s'agit là d'un défaut infinitésimal.

7 Les lecteurs qui ne seraient pas emportés par les thèses ou les hypothèses puissamment avancées par l'auteur devraient au moins lui être reconnaissants de s'être porté au secours d'une anthropologie en danger, et d'avoir fourni pour sa "défense » de nouveaux arguments, et des plus actuels. Parmi ceux-ci, il faut encore signaler le rôle essentiel qu'assigne Maurice Godelier à la critique, non pas seulement dans le principe, mais dans toute la pratique de cette discipline - jusqu'à en faire l'« old mole », la « vieille taupe " animant souterrainement l'ensemble de son ouvrage. Il consacre aussi de nombreuses pages à la réévaluation de "l'imaginaire ", trop peu pris en compte par des recherches récentes ne s'intéressant qu'au «symbolique » ou au "réel », alors que selon lui c'est au contraire dans le premier, l'imaginaire individuel et collectif, que prennent source les représentations susceptibles de fonder des sociétés, dans leur infinie variété. De ce point de vue, ceux qui naguère voulaient porter « l'imagination au pouvoir », en ajoutant : « Soyons réalistes, demandons l'impossible !», seront surpris de se voir révéler ici quarante ans plus tard qu'ils demandaient seulement «l'arrivée de mars en carême ".

8 Mais, s'alarmant de menaces dépassant infiniment celles qui planent sur l'anthropologie - celles qu'«un excès de technique civilisée » et l'obsession du «développement» font peser sur l'environnement mondial, notamment celui du Pacifique auquel est dévolu ce numéro, et même sur l'avenir de l'humanité -, un autre grand océaniste, Gerd Koch, rappelait ce qui constituait à ses yeux la principale leçon de l'anthropologie, "qu'il y avait eu à l'origine un grand nombre de développements possibles dans toutes les directions », faisant mieux comprendre "que c'était la voie 
fausse qui avait été prise", ou plutôt imposée aux autres par l'Occident. Devant l'urgence où se trouve l'espèce humaine de trouver de nouvelles voies "durables » et "soutenables", une anthropologie enfin consciente des pouvoirs et des capacités d'invention de l'imagination humaine, telle que la profile ici Maurice Godelier, serait largement mieux que par le passé en mesure de démontrer sa nécessité et son utilité, tant il apparaît que, dans le « fondement des sociétés », l'enjeu essentiel est leur avenir. 